\title{
Cerrando la brecha de las competencias profesionales genéricas. Un estudio de Teoría Fundamentada
}

\author{
Closing the gap of generic professional skills. A theory based study
}

Adelina Morita Alexander

Universidad Autónoma de Querétaro (UAQ) y Universidad Tecnológica de Querétaro (UTEQ), México.

\section{Alexandro Escudero Nahón \\ Teresa García Ramírez}

Universidad Autónoma de Querétaro (UAQ), México.

\section{Resumen}

Uno de los principales desafíos de las universidades es formar al alumnado con las competencias profesionales que la economía del conocimiento demanda. Las competencias profesionales son competencias específicas de una disciplina y competencias genéricas, que comprenden la correcta gestión de las relaciones interpersonales, del aprendizaje y de los valores sociales. La literatura especializada ha demostrado que los empleadores perciben una brecha entre las competencias genéricas que el mercado laboral requiere y las que los recién egresados de la universidad poseen al ingresar al campo laboral. Sin embargo, no existe tanta literatura que explique cómo "cierran" estos egresados esa brecha. Esta investigación, conducida con la metodología de la Teoría Fundamentada, identificó el proceso informal de aprendizaje que los recién egresados de una institución de educación superior de la ciudad de Querétaro, México, realizaron para ingresar y permanecer en el mercado laboral. En términos generales, adquieren las competencias profesionales genéricas a través de un proceso informal de aprendizaje que consta de cuatro fases: 1) ingreso, 2) permanencia, 3) compromiso y 4) ascenso. En cada una de esas fases las personas aprendieron ciertas competencias profesionales específicas, pero en todas ellas supieron adquirir las genéricas. Este hallazgo permite sugerir que el diseño de un modelo formal de enseñanza de competencias profesionales genéricas para la educación superior debe poner el acento en los procesos informales de aprendizaje, como la observación, la reflexión, la auto regulación y la imitación, pero bajo un proceso estrechamente ligado a los ambientes laborales. Palabras clave: competencias profesionales; empleadores; educación superior; teoría fundamentada.

\begin{abstract}
One of the main challenges of universities is to train students with the professional skills that the knowledge society demands. These professional competences are, on the one hand, specific competences of their discipline, and on the other, generic competences that include the correct management of interpersonal relationships, social learning and social values. The specialized literature has shown that the employers perceive a gap between the generic competences that the labor market requires and the ones that the recent graduates of the university possess when entering the labor field. However, there is not much literature explaining how these newly graduates "close" the gap. This research, conducted with the Grounded Theory, identified the informal learning process that recent graduates of a higher education institution in the city of Querétaro, Mexico, made to enter and remain in the labor market. Generally speaking, they acquire generic professional skills through an informal learning process consisting of four phases: 1)
\end{abstract}


entry, 2) permanence, 3) commitment and 4) promotion. In each of these phases, these people learn certain specific professional competences, but in all of them they know how to acquire the generic ones. This finding suggests that the design of a formal model of generic professional competences for higher education should emphasize informal learning processes, such as observation, reflection, self-regulation and imitation, but under a process closely linked to work environments.

Keywords: professional competences; higher education; employers; grounded theory.

\section{INTRODUCCIÓN}

Una de las características de las sociedades más prósperas es que desde los años setenta del siglo pasado han sustituido paulatinamente sus procesos de producción industrial por una economía basada en el conocimiento para producir capital intangible. Este tipo de economía se caracteriza por producir bienes y servicios a través de actividades que tienen más dependencia de las capacidades intelectuales e interpersonales que de los insumos físicos o los recursos naturales (Švarc \& Dabić, 2015).

La economía basada en el conocimiento provoca que los oficios aislados y de baja interacción, como la agricultura o la manufactura básica, sean subestimados ante profesiones que requieren habilidades y destrezas como la interacción, la resolución de problemas, la habilidad para trabajar en equipos multiculturales, las destrezas digitales, la creatividad, la proactividad, la comunicación asertiva o la correcta gestión de las emociones, entre otras (Dabic, Potocan, \& Nedelko, 2016).

Debido a que lo anterior genera desempleo en amplios sectores de la sociedad, diversos organismos han abordado el desafío de formar a los nuevos profesionales en las competencias necesarias para competir en el mercado actual. En 1998, la Organización de las Naciones Unidas para la Educación, la Ciencia y la Cultura (UNESCO, por sus siglas en inglés), expresó la necesidad de que el aprendizaje fuera considerado un bien permanente para que la población mundial pudiera acceder al mercado laboral. En ese momento el término "competencias profesionales" adquirió una importancia relevante porque impulsó varias iniciativas para contribuir al desarrollo cultural, social y económico de la sociedad (UNESCO, 1998).

El enfoque por competencias profesionales tuvo una amplia y profunda aplicación en las instituciones de educación superior europeas a partir de la Declaración de Bolonia en 1999. Para llevar a cabo lo establecido en esa Declaración, se realizó la iniciativa Tunning Educational Structures in Europe (González \& Wagenaar, 2003), que propuso una metodología para rediseñar, desarrollar, aplicar y evaluar 
los programas de estudio, partiendo de cuatro líneas de acción: 1) Competencias específicas y genéricas, 2) Enfoques de enseñanza, aprendizaje y evaluación, 3) Créditos académicos y 4) Calidad de los programas. Para efectos de este texto, es necesario subrayar que Tunning Educational Structures in Europe ha definido que las competencias profesionales se componen por dos ámbitos: 1) las competencias profesionales especificas, que son conocimientos propios del campo de estudio; y 2) las competencias profesionales genéricas, que están relacionadas con ciertas destrezas "blandas" que permiten aplicar exitosamente los conocimientos en los ambientes laborales en interacción con otras personas (Navío, 2005).

Tunning Europe provocó que América Latina impulsara sus propias experiencias educativas basadas en competencias a través de Tunning América Latina (Beneitone et al, 2007). México participó en el proyecto Tunning América Latina a través del Centro Nacional Tunning, a cargo de la Dirección General de Educación Superior de la Secretaría de Educación Pública (SEP), en colaboración con la Asociación Nacional de Universidades e Instituciones de Educación Superior (ANUIES).

El término competencias se utilizó en un principio en la década de los setenta en el ámbito laboral, asociándolo sobre todo al desarrollo de las habilidades y destrezas que requiere un profesional para demostrar que desempeña bien su labor. Existe un amplio consenso en la academia respecto a que McClelland $(1973,1998)$ realizó los estudios pioneros utilizando este concepto y desde entonces el tema se ha mantenido vigente en el ámbito de la motivación, el desempeño y el rendimiento laboral (Tobón, 2013).

El discurso sobre las competencias ingresó en el ámbito educativo hasta 1980. Pero no fue hasta 1990 que se cristalizó este discurso y comenzaron a surgir modelos educativos basados en competencias adecuados a los diferentes niveles educativos (Argudín, 2006; Fradel, 2009). La Educación Basada en Competencias (EBC) es un término que, aunque se acuñó en instituciones educativas, se ha implementado también en programas de educación no formal (Bellocchio, 2010). Esto fue posible porque la EBC, a diferencia del modelo tradicional de la educación formal, no se basa en el sistema de horas-crédito para obtener grados o certificaciones. De hecho, la EBC se basa en la idea de que el aprendizaje es posible en múltiples escenarios de la vida cotidiana y que el desarrollo de las habilidades, las actitudes y los valores que conforman a las competencias trascienden los límites de tiempo y espacio que suelen caracterizar a la educación tradicional (Bohne, Eicker, \& Haseloff, 2017). 
Actualmente, está ampliamente aceptada la idea de que las competencias profesionales son la combinación dinámica de conocimientos, habilidades, actitudes, saberes, que se integran con base en una serie de atributos personales (capacidades, motivos, rasgos de la personalidad, aptitudes, etcétera) tomando en cuenta las experiencias personales y profesionales. Todo lo anterior se manifiesta a través de ciertos comportamientos o conductas en el ámbito laboral (González \& Wagenaar, 2003; Navío, 2005, pp. 216-217).

Por eso, una correcta formación de competencias profesionales implica, además de la adquisición de conocimientos y habilidades, la capacidad de aplicarlos en la vida real, responder a las demandas en el área ocupacional y llevar a cabo las tareas de forma adecuada (OECD, 2005). El desafío actual es proponer estrategias didácticas que permitan a los alumnos unir dinámicamente la formación técnica y profesional de su ámbito de estudios con ciertas destrezas en el comportamiento social, la actitud para trabajar en equipo, la proactividad, la comunicación asertiva, la capacidad de asumir riesgos, la gestión de las relaciones interpersonales, el aprendizaje y los valores sociales, entre otras (OECD, 2015; Vaca, J., Aguilar, V., Gutiérrez, F., Cano, A. y Bustamante, 2015).

48 Ya se ha mencionado antes en este texto que los expertos de Tunning Educational Structures in Europe destacaron que la línea 1) Competencias específicas y genéricas, era un tema que merecía atención especial para poder garantizar oportunidades de empleo a todas las personas. Esas competencias pueden ser esquematizada de la siguiente manera (González \& Wagenaar, 2003): las competencias específicas son propias del campo de estudio; las competencias genéricas son comunes a cualquier curso de titulación y, a su vez, se clasifican en competencias instrumentales, competencias interpersonales y competencias sistémicas (ver Figura 1).

Las competencias instrumentales son capacidades cognitivas, metodológicas, técnicas y lingüísticas; se consideran necesarias para la comprensión, la construcción, el manejo y el uso crítico de la información en la práctica profesional.

Las competencias interpersonales corresponden a las habilidades de relación social e integración en distintos colectivos, así como la capacidad de desarrollar trabajos en equipos multidisciplinares.

Las competencias sistémicas son habilidades relativas a todos los sistemas, es decir, son una combinación de entendimiento, sensibilidad y conocimiento. 
La literatura especializada en educación acepta que las competencias profesionales específicas y genéricas pueden enseñarse y aprenderse (Luna, 2011; Tobón, 2013; Villa \& Poblete, 2008). Sin embargo, también se ha documentado que las competencias genéricas son las que dotan de éxito profesional y personal a las personas y, curiosamente, suelen aprenderse de manera significativa en ambientes no formales e informales de educación (Escudero, 2013; Kis, 2016).

FIGURA 1

\section{Clasificación de las competencias profesionales}

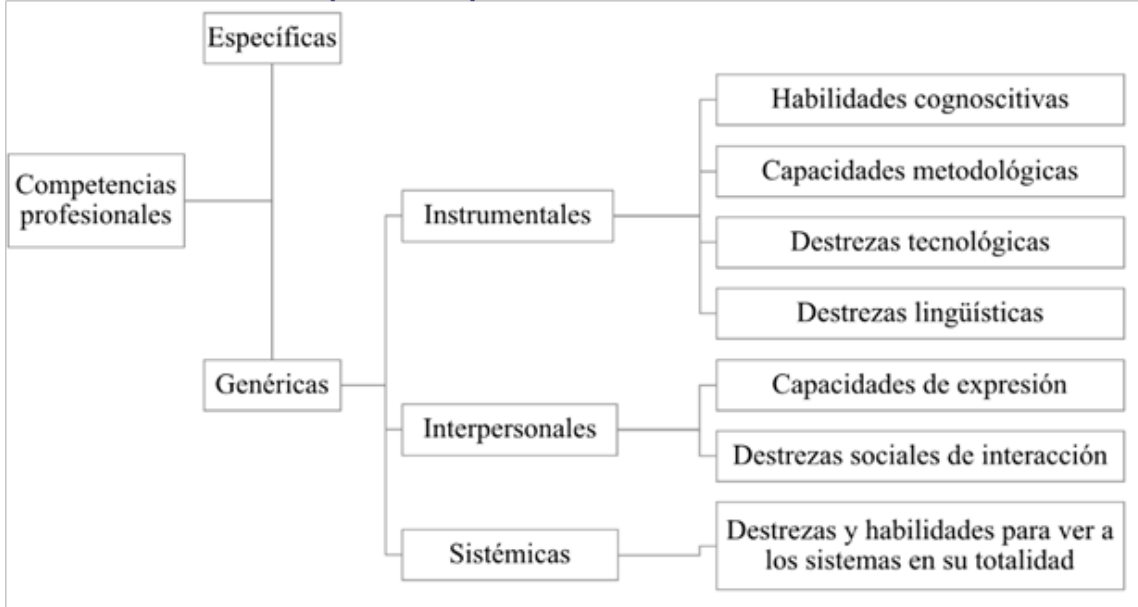

Fuente: (elaboración propia basada en González \& Wagenaar, 2003).

Actualmente el discurso sobre la educación basada en competencias goza de buena credibilidad en América Latina y ha permitido identificar que la falta de competencias profesionales genéricas entre los nuevos profesionales podría estar generando un creciente desempleo, pero irónicamente, las empresas demandan una fuerza laboral bien cualificada para cubrir nuevos puestos (OECD, 2013, 2016a, 2016b).

Esto ha sido demostrado en estudios del Banco de México donde se concluye que en todos los países del mundo el empleo formal del sector industrial, y particularmente del ámbito manufacturero, ha disminuido. En cambio, el empleo en el sector de los servicios ha aumentado y desde el 2008 las oportunidades laborales han crecido más en ocupaciones de alto valor agregado, sobre todo en los países de la OCDE (Alcaraz \& García, 2006)

De tal manera que las economías más avanzadas y las economías en desarrollo, por igual, observan tasas de desempleo elevadas aun cuando existe un número considerable de plazas sin cubrirse (CIDAC, 2014). Esta brecha entre oferta y de- 
manda ha sido señalada principalmente por los empleadores, quienes aseguran que actualmente poseer un título universitario podría ser garantía de la adquisición de competencias profesionales específicas, pero para acceder y desarrollarse exitosamente en el mercado laboral también es necesario demostrar competencias profesionales genéricas.

Debido a eso, algunas instituciones de educación superior comprometidas con la educación de calidad, han intentado institucionalizar estrategias para formar a su alumnado en las competencias profesionales genéricas, posicionando así este tema en el centro de la agenda educativa y motivando estudios al respecto (MengualAndrés, 2013). El equipo de investigación que suscribe este texto ha realizado diagnósticos de percepción y autopercepción sobre competencias profesionales genéricas en instituciones de educación superior en la ciudad de Querétaro, México. Los resultados revelan que existe una diferencia importante entre la percepción de los profesores y la autopercepción de los alumnos respecto al nivel de adquisición de las competencias profesionales genéricas. Los docentes mostraron un punto de vista más optimista sobre el nivel de adquisición de las competencias profesionales genéricas de sus alumnos, mientras que los estudiantes manifestaron que se autoperciben con un bajo nivel de adquisición de estas competencias (Morita, García, \& Escudero, 2016). El mismo estudio identificó que no existen procedimientos institucionalizados para formar las competencias profesionales genéricas en esa institución de educación superior.

Resultados de otros estudios semejantes coinciden en que la educación formal ha creado ciertas experiencias para asumir el reto de formar a los alumnos en las competencias profesionales genéricas, pero aún no existe un modelo generalizado (Marginson, 2010; Weber, 2011). Esta carencia afecta directamente a las empresas porque la innovación y la sustentabilidad necesarias para competir en la economía del conocimiento depende de empleados capaces de solucionar problemas rápida y eficientemente, de gente dispuesta a trabajar en equipos multiculturales, de personas audaces para proponer soluciones creativas de manera asertiva, y sensibles para gestionar adecuadamente las emociones y los ambientes laborales. Por supuesto que también afecta a los recién egresados de las universidades porque quien no posee competencias profesionales genéricas no cuenta con buenas posibilidades para ingresar al mercado laboral o sus expectativas de permanencia y ascenso se reducen considerablemente (Casner-Lotto, J., \& Barrington, 2006). 
Hasta ahora, la formación de competencias profesionales genéricas ha sido solventada principalmente en escenarios de educación no formal, como los programas de educación continua de las empresas. Estudios de opinión realizados a empleadores respecto a la efectividad de la educación continua en las empresas demuestran que es relativamente sencillo actualizar los conocimientos específicos de los profesionales, pero no ha sido tan sencillo dotarlos de competencias profesionales genéricas (CISCO, 2009).

Lo anterior coincide con los resultados de la Encuesta de Competencias de los Adultos, un producto del Programa para la Evaluación Internacional de Competencias de los Adultos (PIAAC, por sus siglas en inglés) donde fue patente que los empleadores valoran los conocimientos técnicos y específicos en sus empleados, como el procesamiento de información con tecnología de punta, o las funciones cognitivas complejas. Sin embargo, consideran fundamental que la fuerza laboral sea capaz de gestionar correctamente las relaciones laborales e interpersonales, el aprendizaje y los valores sociales (Hughes, 2005; OECD, 2015).

Asimismo, la Encuesta de Competencias Profesionales 2014 (ENCOP) realizada por el Centro de Investigación para el Desarrollo A.C. (CIDAC) demostró que 7 de cada 10 empresas mexicanas le asignan mayor importancia a las habilidades "blandas" (personalidad o liderazgo) que a las "duras" (conocimientos específicos) porque, de acuerdo con los empleadores, las competencias "duras" pueden ser formadas con relativa facilidad en programas de educación continua, pero las "blandas" no (CIDAC, 2014).

\section{OBJETIVOS Y MÉTODO DE INVESTIGACIÓN}

Existe una brecha entre las competencias profesionales genéricas que el mercado laboral de la economía del conocimiento requiere y las que los egresados de las instituciones de educación superior poseen al momento de ingresar al campo laboral. Las instituciones de educación superior han iniciado algunos programas para formar las competencias profesionales genéricas entre su alumnado, pero aún no se conoce un modelo generalizado al respecto. Hasta ahora, las empresas han intentado subsanar la falta de competencias profesionales genéricas entre su nueva fuerza laboral a través de programas de educación continua, pero los resultados sugieren que así es más fácil formar competencias profesionales específicas que genéricas. Sin embargo, existen casos de recién egresados que logran ingresar, permanecer e incluso ascender en el mercado laboral. Esta investigación tuvo por 
objetivo identificar cómo logran estas personas "cerrar" exitosamente la brecha entre las competencias profesionales genéricas que el mercado demanda y las que ellas poseen al egresar de los estudios universitarios.

La educación formal no ha demostrado ser un lugar privilegiado para la formación de las competencias profesionales genéricas (Boni \& Lozano, 2007; Kallioinen, 2010). Al contrario, la literatura especializada sobre educación basada en competencias ha llamado la atención sobre el hecho de que el contexto tiene cierta importancia en el proceso de adquisición de las competencias profesionales genéricas, pero ya sea porque no existen contextos educativos institucionales que las fomenten sistemáticamente o porque este tipo de competencias se aprenden mejor por vías informales, el resultado es que los procesos individuales son definitorios para su aprendizaje (Renta Davids, van Den Bossche, Gijbels, \& Fandos Garrido, 2016).

El desafío de formar a las personas en las competencias profesionales genéricas requeridas en la economía del conocimiento es relevante para las universidades. Sin embargo, debido a que son los empleadores quienes se ven obligados a solventar esa carencia en su fuerza laboral, el tema ha sido más estudiado en los ambientes laborales. Existe abundante literatura sobre los programas de educación continua que las empresas formalizan para actualizar a sus empleados en las competencias profesionales específicas, es decir, en los conocimientos propios de su disciplina.

Sin embargo, no existe tanta literatura que explique cuáles son los principales problemas a los que se enfrentan los recién egresados de las instituciones de educación superior y cómo los resuelven en el ámbito laboral. Puesto que el objetivo de esta investigación fue identificar cómo, aun sin intermediación institucional, algunos egresados de las universidades han sido capaces de "cerrar" exitosamente esa brecha, era necesario conducir el estudio con un método de investigación capaz de rastrear las acciones de los participantes. El método más adecuado para tal fin fue la Teoría Fundamentada.

La investigación se condujo con el método de la Teoría Fundamentada porque es un procedimiento capaz de obtener información y analizarla simultáneamente de manera inductiva-abductiva (Bryant, 2017). El objetivo de la Teoría Fundamentada es colaborar en la formación de teorías de rango medio que puedan explicar el principal problema de cierto grupo de personas y las estrategias que realizan para solucionarlo (Gibson \& Hartman, 2014; Holton \& Walsh, 2016). 
A diferencia de los estudios hipotético-deductivos, la Teoría Fundamentada ingresa al campo de estudio sin categorías de análisis preconcebidas. Al contrario, construye las categorías de análisis con un proceso recursivo de obtención de datos, codificación de datos e interpretación (ver Tabla 1).

\section{TABLA 1}

\section{Diferencias entre el proceso de investigación hipotético-deductiva y la Teoría Fundamentada}

\begin{tabular}{|l|l|}
\hline \multicolumn{1}{|c|}{ Investigación hipotético-deductiva } & \multicolumn{1}{c|}{ Teoría Fundamentada } \\
\hline $\begin{array}{l}\text { Revisión de literatura y elaboración del marco } \\
\text { teórico }\end{array}$ & $\begin{array}{l}\text { Preguntarse "¿Cuál es el principal problema de este } \\
\text { grupo de personas y qué hacen para resolverlo?" }\end{array}$ \\
\hline $\begin{array}{l}\text { Definición precisa de la pregunta o problema de } \\
\text { investigación }\end{array}$ & $\begin{array}{l}\text { Recogida de datos y análisis simultáneo de los } \\
\text { mismos }\end{array}$ \\
\hline Planteamiento de la(s) hipótesis & Construcción de Códigos y Categorías Analíticas \\
\hline Descripción de los objetivos de la investigación & Método Comparativo Constante y Muestreo Teórico \\
\hline Recogida de datos & $\begin{array}{l}\text { Definición de la Unidad de Análisis y Muestreo } \\
\text { Teórico Dirigido }\end{array}$ \\
\hline Análisis e interpretación de los datos & $\begin{array}{l}\text { Revisión de literatura y explicación del problema } \\
\text { con base en una categoría de análisis construida } \\
\text { en el campo de estudio }\end{array}$ \\
\hline Redacción del informe & Redacción del informe \\
\hline
\end{tabular}

Fuente: (Escudero, 2014).

El procedimiento general se basa en obtener datos de varias fuentes de información y codificarla en tres fases: codificación abierta (para empezar a construir categorías descriptivas), codificación axial (para ordenar estas categorías en torno a una categoría conceptual central), y finalmente una codificación teórica, también llamada conceptualización (para desarrollar un orden de ideas que expliquen el problema) (Bryant \& Charmaz, 2010).

Puesto que el objetivo de un estudio de Teoría Fundamentada no es verificar una hipótesis, sino explicar cuál es el principal problema de un grupo de personas y qué hacen para solucionarlo, es necesario construir una categoría de análisis explicativa. Por eso, la muestra de este tipo de estudios no es una muestra representativa, sino una muestra teórica. Es decir, se consulta la cantidad de personas necesarias para saturar las propiedades y dimensiones que permitan construir una categoría analítica. 


\subsection{Procedimiento de obtención de datos}

Para la obtención de datos se realizaron dos grupos de enfoque, uno con empleadores y el otro con egresados, con la finalidad de identificar las carencias en relación a la formación en competencias profesionales, al que se han enfrentado los empleadores y los egresados. La pertinencia de usar el grupo de enfoque en este estudio radica en los fundamentos teóricos que han demostrado que dicha técnica recoge información a partir de experiencias, opiniones y expectativas de grupos bien delimitados, situación que puede extrapolarse con cierta facilidad a grupos semejantes de la sociedad (Monje, 2011).

Para la selección de la muestra se buscó la participación de informantes clave (sujetos tipo) que reunieron las condiciones y características necesarias para la investigación en ambos grupos de enfoque.

El proceso de selección de los participantes del grupo de empleadores constó de las siguientes etapas:

a) Se enviaron 100 correos electrónicos a empleadores solicitando su participación en la investigación. Los correos se dirigieron a empresarios del sector público y/o privado que han proporcionado trabajo a egresados de la universidad y que ocupan puestos a nivel gerencial.

b) Vía correo electrónico se obtuvo respuesta positiva de 25 empleadores que proporcionaron su número telefónico a manera de confirmación.

c) Se les informó sobre los detalles del grupo de enfoque (lugar, fecha y hora). En esta etapa solo 15 empleadores mostraron disposición para participar.

d) Se envió invitación personalizada vía correo electrónico a los 15 empleadores que confirmaron asistencia.

e) Finalmente a este grupo de enfoque asistieron 8 empleadores (ver Tabla 2).

Para el grupo de enfoque con egresados se siguió el siguiente procedimiento:

a) Se contactó vía Facebook® a 200 egresados que pertenecen a las generaciones 2013, 2014 y 2015 cuyo plan de estudios estuvo bajo el enfoque por competencias profesionales, invitándolos a participar en la investigación. En esta etapa 50 egresados mostraron interés.

b) Se pidió a los interesados enviar mensaje privado con número de celular y un correo electrónico para confirmar.

c) C) Se envió invitación personalizada vía correo electrónico a los 30 egresados que proporcionaron la información solicitada, con información de lugar, fecha y hora del grupo de enfoque. 
d) D) Se recibieron 12 respuestas confirmando asistencia.

e) E) Se contó con la asistencia y participación de 7 egresados de la universidad, de los cuales dos corresponden a la generación 2013, dos a la 2014 y 3 a la 2015.

TABLA 2

Características de las empresas participantes

\begin{tabular}{|c|c|c|c|}
\hline Número de participantes & Tamaño & Sector económico & Capital \\
\hline 1 & Micro & Servicios & Privado \\
\hline 3 & Pequeña & Industria & Privado \\
\hline 2 & Mediana & Servicios & Público \\
\hline 2 & Grande & Servicios & Público \\
\hline
\end{tabular}

Fuente: elaboración propia.

El procedimiento para planear, diseñar, desarrollar y analizar los grupos de enfoque fue conducido de acuerdo a la literatura especializada (Cataldi, 2017; Shepherd, 2016; Tadajewski, 2016)this study will use the relational analysis, applying it to two specific families of interrogation techniques: the focus groups and the in-depth interviews. Therefore, a pilot study is conducted analysing a small number of focus group transcriptions and in-depth interviews on the base of the Interaction Process Analysis (Bales in Interaction process analysis: a method for the study of small groups, Chicago University Press, Chicago, 1950 (ver Figura 2).

\section{FIGURA 2}

Procedimiento de planeación, diseño, desarrollo y análisis de los grupos de enfoque

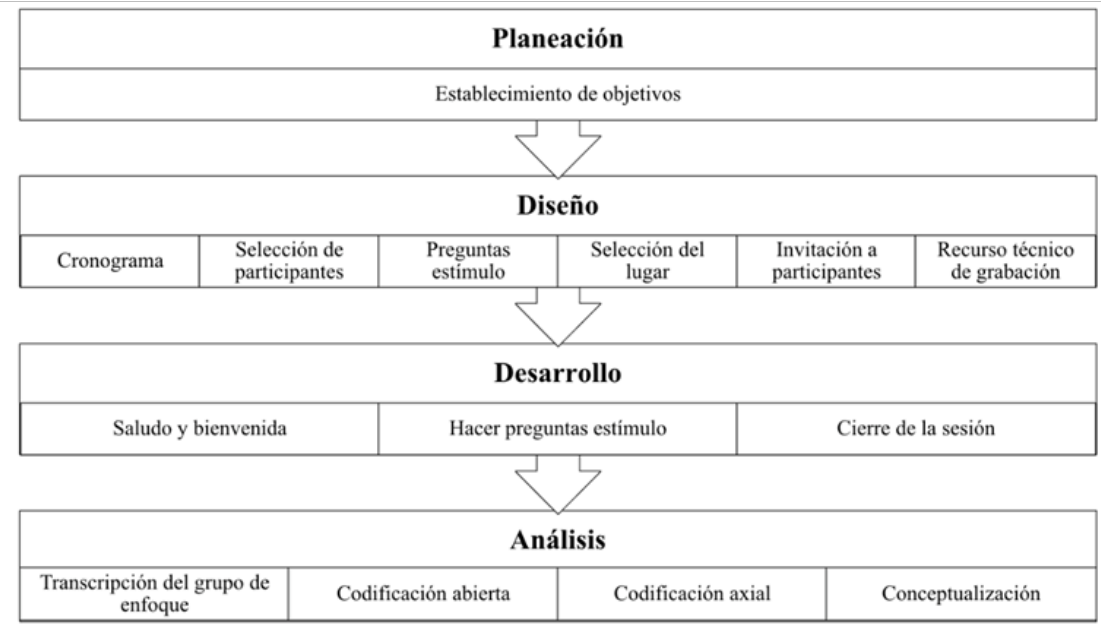

Fuente: (elaboración propia basada en Stewart \& Shamdasani, 2014). 


\subsection{Análisis de los datos}

Se realizaron los grupos de enfoque con empleadores y con egresados, en diferentes fechas y lugares. Cada grupo de enfoque tuvo una duración aproximada de dos horas. Las participaciones fueron grabadas en audio y posteriormente se realizó la transcripción.

Las fases de codificación abierta, axial y teórica (conceptualización), se realizaron a partir de la información obtenida de las siguientes preguntas desencadenantes para empleadores:

- ¿Qué les falta a los alumnos recién egresados de la carrera de Administración de la universidad para desempeñarse adecuadamente en el ambiente laboral?

- ¿Qué hicieron para resolverlo?

Y de las preguntas estímulo para egresados:

- ¿Cuál fue su principal problema al ingresar al campo laboral?

- ¿Qué hicieron ustedes para resolverlo?

56 Simultáneamente a la codificación se realizaron memorándums para ir construyendo categorías de análisis para explicar las respuestas anteriores (ver Anexo B).

A continuación se describe cada una de las tres fases de codificación.

\subsubsection{Codificación abierta}

Para lograr la codificación abierta fue necesario: 1) transcribir completamente las declaraciones de los miembros de los grupos de enfoque; 2 ) identificar fragmentos de información relevante y adjudicarle un código (ver Anexo A); 3) diseñar memorándums (ver Anexo B) que diesen cuenta de la manera en que surgen las categorías analíticas, cómo evolucionan y cómo impulsan el avance de la investigación (Gibson \& Hartman, 2014).

Asimismo, se identificaron dos tipos de códigos: los códigos "construidos", que son propuestas conceptuales del equipo de investigación; y los códigos "en vivo", que son expresiones que las personas entrevistadas expresaron como tal (en cursiva). La Tabla 3 y la Tabla 4 se presentan las listas de códigos obtenidas a partir de las preguntas estímulo. 


\section{TABLA 3}

Lista de códigos del grupo de enfoque a empleadores de la pregunta: "¿qué les falta a los alumnos recién egresados de la carrera de Administración para desempeñarse adecuadamente en el ambiente laboral?"

No dan retroalimentación al director.

Piensan: no me meto para que no me den más chamba.

Innovar implica ser creativo.

La dirección requiere saber de todo.

No hay compromiso con la empresa.

Falta sentido de pertenencia.

No hay plan de vida.

La empresa subestima la responsabilidad de enseñarles.

Los egresados representan muchas áreas de oportunidad.

Que el título TSU no sea limitativo.

Si la persona es capaz, va a llegar.

El programa educativo puede ser robusto.

Les falta conocimiento sobre cómo desarrollarse en el ambiente laboral.

Necesitan comunicación y autoconfianza.

Si no logran encajar en la primera semana no van a encajar nunca.

Tiene buena formación, pero no la saben aplicar en la empresa.

Necesitan autoestima.

Necesitan liderazgo.

Mejorar uso de las TIC

Necesitan comunicación asertiva.

Necesitan empoderarse.

Necesitan romper sus autolimitaciones.

Falta capacidad para comunicación oral y escrita.

Necesitan autoestima y confianza.

\section{Tabla 4}

Lista de códigos del grupo de enfoque a empleadores de la pregunta: "¿qué hacen para ayudarlos a resolverlo?"

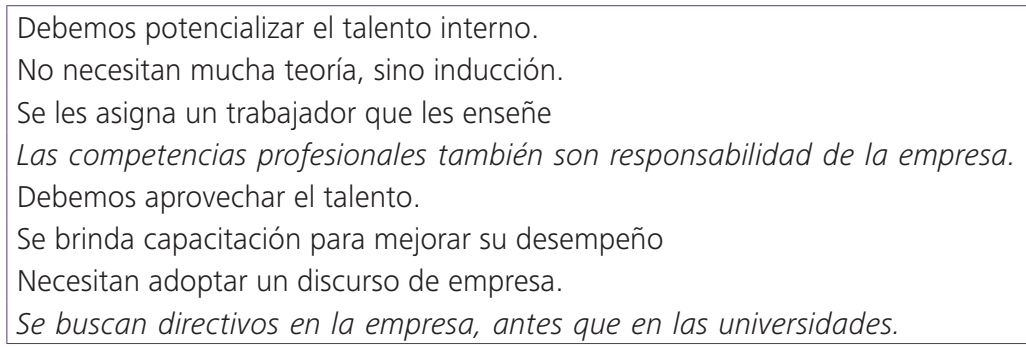

La Tabla 5 presenta la lista de códigos obtenida en el grupo de enfoque de egresados de la UTEQ a partir del tema: "¿cuál fue su principal problema al ingresar al campo laboral?". 


\section{TABLA 5}

\section{Lista de códigos del grupo de enfoque a egresados de la primer pregunta estímulo}

El horario es incompatible.

Salario bajo.

Solicitan experiencia a los recién egresados.

Las necesidades laborales no corresponden a los conocimientos de la universidad.

La escuela es para teoría, el trabajo para la práctica.

Debemos conocer las competencias de nuestra profesión.

Profesores como contacto hacia las empresas.

La escuela ofrece algo, el individuo define su trayectoria.

saber venderte.

Los profesores deben saber qué requiere el mercado laboral.

Las estadías no están sistematizadas.

Empresas requieren conocimientos en psicología laboral.

Prácticas reales en la escuela.

El trabajo en equipo no forma para el trabajo.

La escuela debería actualizar su enseñanza de software.

Se debería actualizar el plan de estudios.

Falta conocer los programas usados en las empresas

Comunicación en un segundo idioma

Una vez adentro ya me empiezo a mover.

Tener competencias digitales.

Si sabes y te gusta, vas a aprender.

58 Hace falta seguridad.

La escuela forma el criterio.

Capacidad de comunicación asertiva

Dominar un segundo idioma

Habilidades en el uso de las TIC

El trabajo en equipo es como un cáncer para nosotros como alumnos.

No es lo mismo trabajo colaborativo que trabajo en equipo.

Conocer las competencias propias.

Tenemos conocimientos, pero nos falta saber aplicarlos.

Falta capacidad para resolver problemas.

Falta capacidad para comunicación oral y escrita.

Hace falta liderazgo.

\section{TABLA 6}

\section{Lista de códigos del grupo de enfoque a egresados, de la segunda pregunta estímulo}

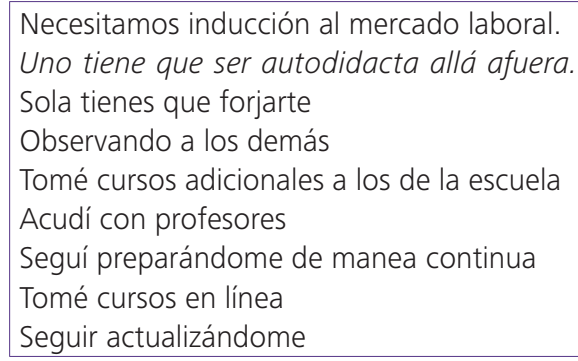




\subsubsection{Codificación axial}

A partir de los códigos obtenidos en la codificación abierta se realizaron tres acciones: 1) una definición de la(s) categoría(s) principal(es) y de las periféricas, 2) la identificación de las competencias profesionales genéricas que cada grupo señaló como competencias que requieren desarrollar (se observó coincidencia en algunas competencias); y, 3) un ordenamiento analítico y dinámico entre las categorías para identificar un patrón o un proceso relevante.

Existe una brecha entre lo que saben y lo que deben saber resultó ser la categoría axial. Esta categoría hace alusión a que las competencias profesionales genéricas no se adquieren a través de la educación formal de los estudios superiores, pero son cruciales para ingresar, permanecer y ascender en el campo laboral (ver Figura 3).

Las categorías periféricas gravitan alrededor de la categoría axial describiendo el siguiente patrón: los recién egresados que pueden adquirir las competencias profesionales genéricas en el ambiente laboral logran permanecer en la empresa. Si no se adquieren las competencias genéricas en la empresa, tarde o temprano se produce el despido. Los procesos a través de los cuales se aprenden las competencias profesionales genéricas son principalmente por imitación de un modelo profesional exitoso de la empresa. Tras identificar a un profesional exitoso en la empresa, el recién egresado observa, reflexiona e imita las actitudes profesionales genéricas del modelo.

\section{FIGURA 3}

\section{Categoría axial y periféricas}

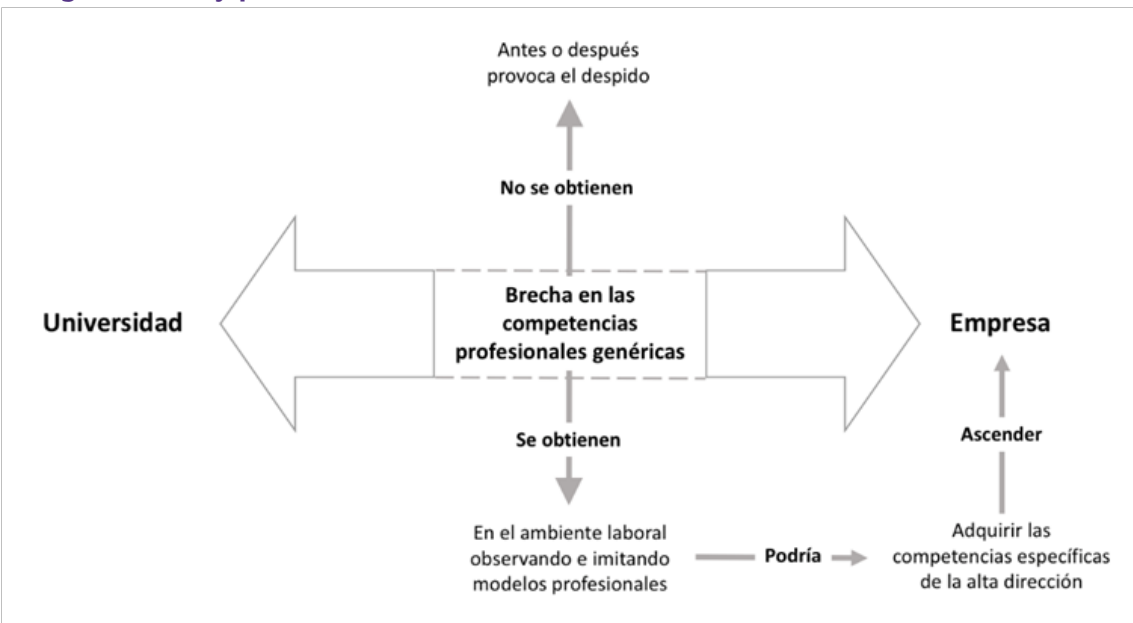

Fuente: elaboración propia. 
Se realizó una representación gráfica de las competencias profesionales genéricas que los empleadores consideran que deben ser fortalecidas en los recién egresados de las instituciones de educación superior. Al mismo tiempo, se identificó cuáles de estas competencias creen los recién egresados que deben fortalecer ellos mismos. En la Figura 4 se muestra este ámbito de intersección.

Aunque solo tres competencias se localizan en el ámbito de intersección, la importancia de este hallazgo radica en que la mayoría de las competencias que deben ser fortalecidas, según la opinión de empleadores o según la opinión de los recién egresados, son en realidad competencias profesionales genéricas.

Destaca el hecho de que varias de esas competencias estén relacionadas con puestos de mandos medios o gerenciales (ver Figura 4). De manera que la codificación axial sí es congruente con los estudios previos respecto a la peculiar situación de desempleo y oferta de empleo en la economía del conocimiento.

\section{FIGURA 4}

\section{Opinión de empleadores y recién egresados sobre las competencias genéricas que} deberían ser fortalecidas

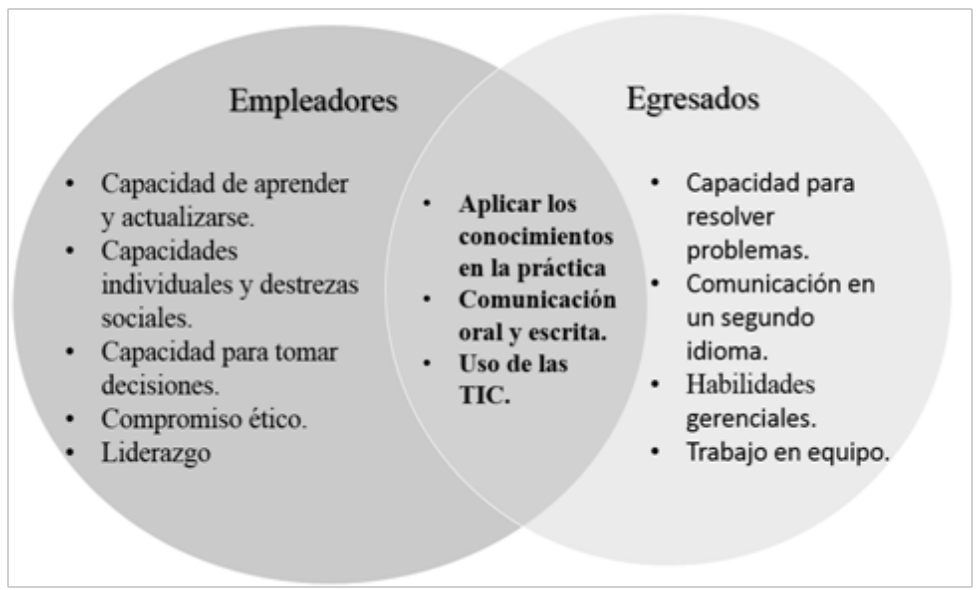

Fuente: elaboración propia.

\subsubsection{Conceptualización}

En la codificación teórica, o conceptualización, fue posible explicar cuál es el principal problema de los recién egresados al ingresar al ámbito laboral y qué hacen para resolver ese problema. 
En primer lugar, se ha identificado que el principal problema de los recién egresados al ingresar al ámbito laboral es demostrar competencias profesionales genéricas, pero uno de los hallazgos de este estudio es haber identificado que estas competencias profesionales genéricas están relacionadas con otro tipo de competencias que no han sido mencionadas en la literatura especializada.

Hemos titulado a estas competencias como competencias profesionales específicas abstractas y competencias profesionales específicas técnicas (ver Figura 5).

\section{FIGURA 5}

\section{Clasificación de las competencias profesionales específicas (abstractas y técnicas)}

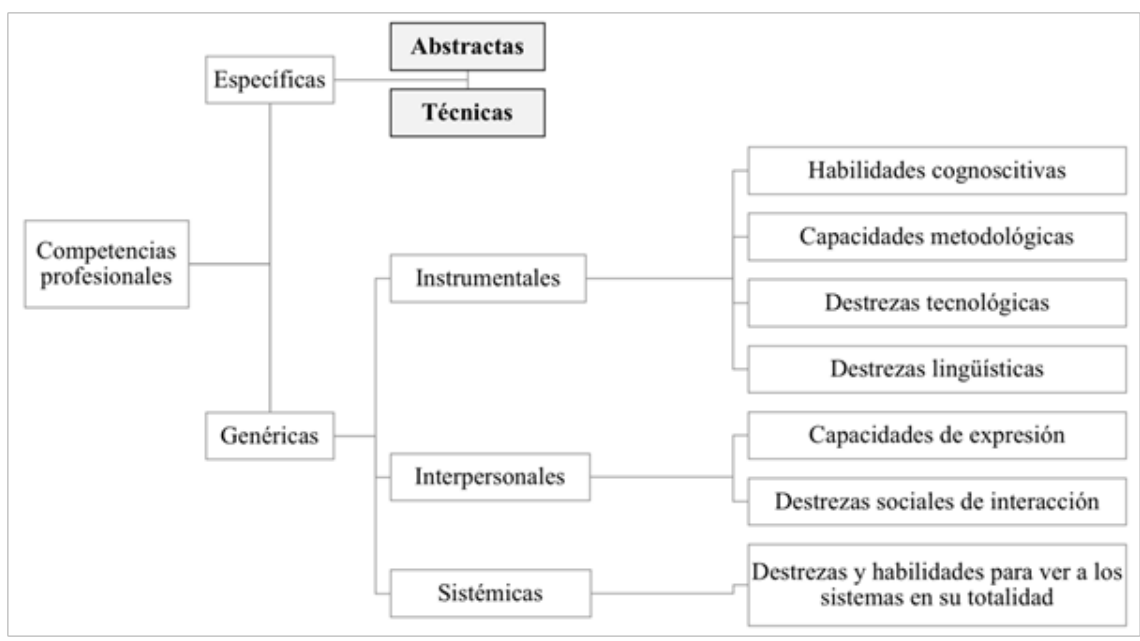

Fuente: elaboración propia.

Las competencias profesionales específicas abstractas son conocimientos disciplinares que están íntimamente relacionados a los cargos de alta dirección, mientras que las competencias profesionales específicas técnicas se aplican en los niveles operativos. Al hacer esta distinción entre competencias profesionales específicas abstractas y competencias profesionales específicas técnicas es posible entender qué relación guardan las competencias profesionales en relación a los niveles de gestión de las empresas (ver Figura 6). 
FIGURA 6

\section{Competencias profesionales requeridas según nivel de gestión en la empresa}

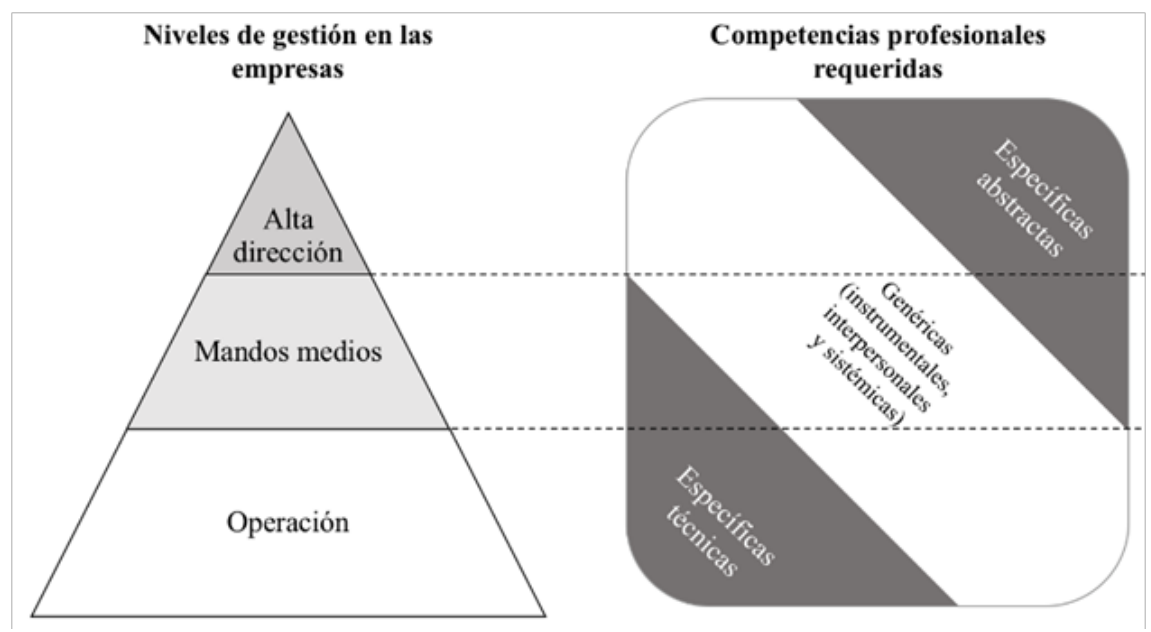

Fuente: elaboración propia.

Así, es posible demostrar que las competencias profesionales genéricas sí son necesarias en todos los niveles de gestión de las empresas, pero sobre todo, es posible describir el proceso a través del cual algunos recién egresados logran ingresar, permanecer y ascender en el ámbito laboral.

Por eso, en segundo lugar, la conceptualización ha permitido describir el proceso de aprendizaje informal de competencias a través del cual los recién egresados logran permanecer y ascender en el ámbito laboral. Dicho proceso consta de cuatro fases: ingreso, permanencia, compromiso y ascenso (ver Figura 7).

1. Ingreso. Se ha mencionado antes que existe una brecha entre lo que saben y lo que deben saber es una categoría de análisis que puede explicar el hecho de que los recién egresados acceden al campo laboral con ciertos conocimientos, habilidades y actitudes que corresponden a las competencias específicas de su profesión, pero generalmente no cumplen con las expectativas que los empresarios tienen sobre las competencias profesionales genéricas. La Figura 7 muestra que en la fase de ingreso algunos recién egresados suelen superar esta brecha demostrando tener muy buenas competencias profesionales específicas técnicas. 


\section{FIGURA 7}

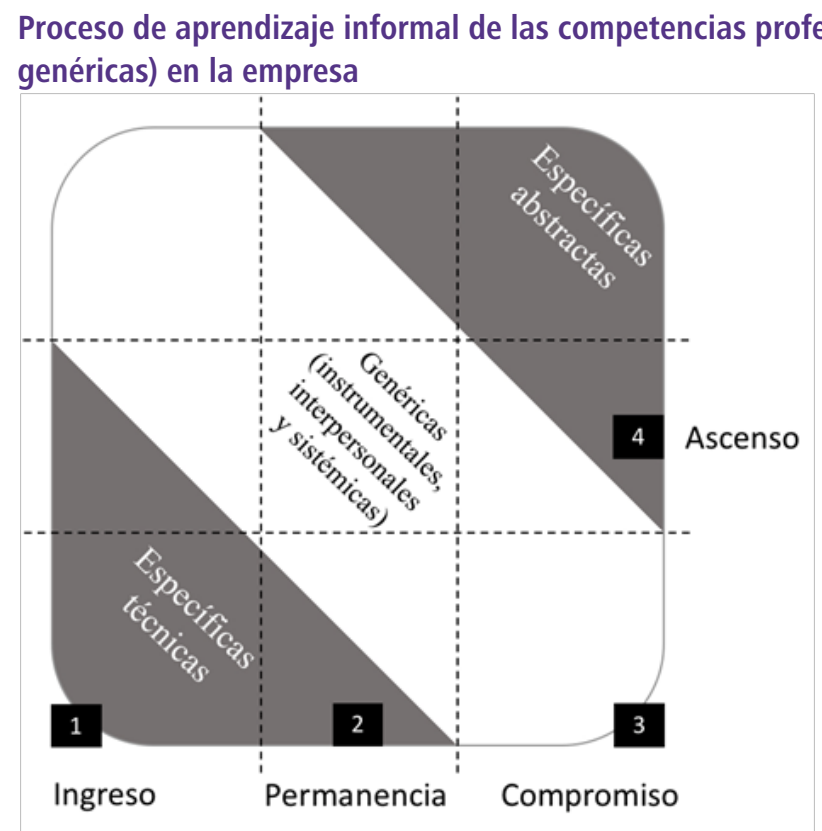

Fuente: elaboración propia.

2. Permanencia. La brecha entre las competencias profesionales específicas y genéricas suele ser abordada por los empleadores con la aplicación de programas de educación continua al interior del ámbito laboral. No obstante, estos programas suelen tener más éxito para formar competencias profesionales específicas que genéricas. Los recién egresados manifestaron que una estrategia que utilizan para adquirir competencias genéricas es observar la manera en que se conducen los profesionales bien valorados de la empresa. Ese aprendizaje por "imitación" es informal, espontáneo y se autorregula dinámicamente. Respecto a esta fase, los empleadores comentaron que las personas que demuestran cierto potencial en la empresa son apoyados con capacitación específica para que su desempeño mejore. Pero este estudio identificó que la manera de demostrar "potencial" es una destreza de las competencias genéricas, pues supone simpatía, empatía, responsabilidad, compromiso, proactividad... Se puede deducir que solamente aquellos que son capaces de adquirir las competencias profesionales genéricas al mismo tiempo que afinan las competencias profesionales específicas de índole técnica, tienen altas posibilidades de permanecer en el ámbito laboral. 
3. Compromiso. Este estudio de Teoría Fundamentada obtuvo datos empíricos respecto al hecho de que los empleadores sí están dispuestos a promover las trayectorias profesionales de su fuerza laboral, siempre y cuando los empleados demuestren tener cierto compromiso con la empresa. Incluso están dispuestos a colaborar en programas de vinculación entre universidades y empresas porque admiten que requieren el talento de los universitarios para competir en la economía del conocimiento. Sin embargo, a cambio requieren estar seguros que los empleados susceptibles de ser promovidos tienen un compromiso estrecho con la empresa. Una vez más, la manera de demostrar ese compromiso requiere más competencias profesionales genéricas que específicas porque se hace patente en la proactividad, el liderazgo o la capacidad de generar ambientes productivos. Saber demostrar estas competencias es la antesala del ascenso laboral y, de acuerdo con los resultados de este estudio, estas competencias difícilmente se enseñan, pero sí se aprenden por vías informales.

4. Ascenso. De acuerdo con la información obtenida, los empresarios aseguran que para que una persona ascienda laboralmente debe demostrar su compromiso con la empresa, pero no es suficiente esa situación. Además, debe adquirir nuevas competencias profesionales específicas de los mandos medios que le permitan realizar actividades gerenciales. Por eso, la Figura 7 muestra que esta fase comprende un aprendizaje informal de ciertas competencias profesionales genéricas, pero es obligado ingresar a programas educativos formales o no formales para adquirir ciertas competencias profesionales específicas abstractas.

\section{CONCLUSIONES}

La economía del conocimiento ha planteado varios desafíos a la educación superior porque la fuerza laboral capaz de ingresar y ascender en los ámbitos laborales actualmente requiere cierto tipo de competencias que no suelen aprenderse en la educación formal. Estas son las competencias profesionales genéricas, que comprenden la correcta gestión de las relaciones interpersonales, del aprendizaje y de los valores sociales.

Las competencias profesionales genéricas son imprescindibles para lograr una estabilidad en las empresas, pero también son condición de posibilidad para el ascenso profesional. Las personas que adquieren competencias profesionales genéricas suelen adquirir paulatinamente más responsabilidades laborales, mejores perspectivas profesionales y económicas. 
De acuerdo con la literatura especializada, los empleadores perciben una brecha entre las competencias genéricas que el mercado laboral requiere y las que los recién egresados de la universidad poseen al ingresar al campo laboral. Sin embargo, existen algunos casos de éxito de recién egresados de las universidades que sí son capaces de ingresar, permanecer y ascender en las empresas.

Esta investigación, conducida con la metodología de la Teoría Fundamentada, identificó que algunas personas son capaces de aprender las competencias profesionales genéricas en los ambientes laborales, de manera informal y a través de cuatro fases: ingreso, permanencia, compromiso y ascenso.

En cada una de estas fases las personas aprenden competencias profesionales específicas, pero en todas ellas deben ir incorporando competencias profesionales genéricas. El ascenso se obtiene cuando el empleado es capaz de demostrar adecuadamente que posee estas competencias genéricas y que está dispuesto a aprender competencias profesionales específicas de los mandos gerenciales.

Aunque actualmente no existen modelos educativos formales que ayuden a los estudiantes a adquirir las competencias profesionales genéricas que se requieren para tener éxito en la economía del conocimiento, sí existe disposición por parte de las empresas para institucionalizar vínculos con las universidades y participar así en los nuevos diseños curriculares.

No obstante, en esta ocasión hay mucho que aprender de las personas que, sin ayuda de las instituciones de educación superior, han sabido adquirir a través de la observación, la reflexión, la auto regulación y la imitación, competencias profesionales genéricas. Es necesario realizar más investigación empírica al respecto.

\section{REFERENCIAS}

Alcaraz, C., \& García, R. (2006). Cambios en la Composición del Empleo y Evolución de la Productividad del Trabajo en el Sector Formal de la Economía Mexicana: 2000-2005 (Documentos de Investigación No. 2006-3). Ciudad de México. Recuperado a partir de http://www.banxico.org.mx/publicaciones-y-discursos/ publicaciones/documentos-de-investigacion/banxico/\%7B4E97827E-F364-21D7B392-B223BC039B0C\%7D.pdf

Argudín, Y. (2006). Educación Basada en Competencias: antecedentes y nociones. México: Trillas. 
Bellocchio, M. (2010). Educación Basada en Competencias y constructivismo: un enfoque y un modelo para la formación pedagógica del siglo XXI. México: Cuaderno de casa ANUIES.

Beneitone, P., Esquetini, C., González, J., Maletá, M., Siufi, G., \&Wagenaar, R. (2007). Reflections on and outlook for Higher Education in Latin America. Final Report-Tunning Latin America Project 2004-2007. Bilbao: Universidad de Deusto. Recuperado a partir de http://www.unideusto.org/tuningeu/images/stories/Publications/ Tuning_A_Latina_INGL_PR2.pdf

Bohne, C., Eicker, F., \& Haseloff, G. (2017). Competence-based vocational education and training (VET): An approach of shaping and networking. European Journal of Training and Development, 41(1). https://doi.org/10.1108/EJTD-07-2016-0052

Boni, A., \& Lozano, J. F. (2007). The generic competences: An opportunity for ethical learning in the European convergence in higher education. Higher Education, 54(6), 819-831. https://doi.org/10.1007/s10734-006-9026-4

Bryant, A. (2017). Grounded Theory and Grounded Theorizing: Pragmatism in Research Practice. New York: Oxford University Press.

Bryant, A., \& Charmaz, K. (2010). The SAGE handbook of grounded theory. Los Angeles: SAGE.

Casner-Lotto, J., \& Barrington, L. (2006). Are They Really Ready to Work? Employers' Perspectives on the Basic Knowledge and Applied Skills of New Entrants to the 21st Century US Workforce. Recuperado a partir de http://www.p21.org/ storage/documents/FINAL_REPORT_PDF09-29-06.pdf

Cataldi, S. (2017). A proposal for the analysis of the relational dimension in the interview techniques: a pilot study on in-depth interviews and focus groups. Quality and Quantity. https://doi.org/10.1007/s11135-017-0468-9

CIDAC. (2014). Encuesta de competencias profesionales 2014. Ciudad de México. Recuperado a partir de http://cidac.org/esp/uploads/1/encuesta_competencias_ profesionales_270214.pdf

CISCO. (2009). Preparar a cada alumno para el siglo XXI. Recuperado a partir de http://www.cisco.com/c/dam/en_us/about/citizenship/socio-economic/docs/ GlobalEdWPLatAm.pdf

Dabic, M., Potocan, V., \& Nedelko, Z. (2016). Personal values supporting enterprises' innovations in the creative economy. Journal of the Knowledge Economy, 1-21. https://doi. org/10.1007/s13132-016-0354-z

Escudero, A. (2013). "Responsible trust": A value to strengthen Active Citizenship. Journal of Social Studies Education Research, 4(2), 27-54. Recuperado a partir de http:// www.jsser.org/index.php/JSSER/article/viewFile/148/pdf

Escudero, A. (2014). Identidades e Identificaciones para la cohesión social: Estudio de Teoría Fundamentada sobre programas de voluntariado. En XIV Congreso de Investigación sobre el Tercer Sector. Innovación social y redes de sociedad civil en las agendas global y local. México: Universidad Anáhuac del Norte. 
Fradel, L. (2009). Desarrollo de competencias en educación: desde preescolar hasta el bachillerato. México: Inteligencia educativa.

Gibson, B., \& Hartman, J. (2014). Rediscovering grounded theory. Beverly Hills (California) [etc.]: Sage.

González, J., \& Wagenaar, R. (2003). Tunning Educational Structures in Europe. Bilbao. Recuperado a partir de http://www.unideusto.org/tuningeu/

Holton, J., \& Walsh, I. (2016). Classic grounded theory: applications with qualitative and quantitative data. Thousand Oaks, Calif., Calif.: Sage Publications.

Hughes, J. (2005). Bringing emotion to work: emotional intelligence, employee resistance and the reinvention of character. Work Employment \& Society, 19(3), 603-625.

Kallioinen, O. (2010). Defining and comparing generic competences in higher education. European Educational Research Journal, 9(1), 56-68. https://doi.org/10.2304/ eerj.2010.9.1.56

Kis, V. (2016). Work, train, win: work-based learning design and management for productivity gains (OECD Education Working Papers No. 135). Paris. https:// doi.org/http://dx.doi.org/10.1787/5jlz6rbns1g1-en

Luna, M. E. (2011). Elementos contextuales que influyen en los aprendizajes y el desarrollo de competencias. En La formación cívica y ética en la educación básica: retos y posibilidades en el contexto de la sociedad globalizada (pp. 67-83). México: SEP.

Marginson, S. (2010). Higher education in the global knowledge economy. Procedia Social and Behavioral Sciences, 2(5), 6962-6980. https://doi.org/10.1016/j. sbspro.2010.05.049

McClelland, D. C. (1973). Testing for competence rather than intelligence. American Psychologist, 28, 1-14.

McClelland, D. C. (1998). Identifying competencies with behavioral event interviews. Psychological Science, 9(5), 331-339.

Mengual-Andrés, S. (2013). Rethinking the role of Higher Education. New Approaches in Educational Research, 2(1), 1-2. https://doi.org/10.7821/naer.2.1.1-2

Monje, C. (2011). Metodología de la investigación cuantitativa y cualitativa. Colombia: Facultad de Neiva.

Morita, A., García, M. T., \& Escudero, A. (2016). Análisis de la percepción de las competencias genéricas en instituciones de educación superior en México. Revista de Educación y Desarro/lo, 38, 69-78. Recuperado a partir de http://www.cucs.udg.mx/revistas/ edu_desarrollo/anteriores/38/38_Morita.pdf

Navío, A. (2005). Propuestas conceptuales en torno a la competencia profesional. Revista de Educación, 337, 213-234.

OECD. (2005). The definition and selection of key competencies. Executive summary. 
OECD. (2013). OECD Skills Outlook 2013: First Results from the Survey of Adult Skills. Paris: OECD Publishing. https://doi.org/10.1787/ 9789264204256-en

OECD. (2015). Skills for Social Progress: The Power of Social and Emotional Skills. Paris: OECD Skills Studies Publishing. https://doi.org/10.1787/9789264226159-en

OECD. (2016a). OECD Employment Outlook 2016. Paris: OECD Publishing. https://doi. org/10.1787/empl_outlook-2016-en

OECD. (2016b). Skills Matter: Further Results from the Survey of Adult Skills. Paris: OECD Publishing. https://doi.org/http://dx.doi.org/10.1787/9789264258051-en

Renta Davids, A. I., van Den Bossche, P., Gijbels, D., \& Fandos Garrido, M. (2016). The Impact of Individual, Educational, and Workplace Factors on the Transfer of School-Based Learning into the Workplace. Vocations and Learning. https://doi.org/10.1007/ s12186-016-9168-1

Shepherd, G. (2016). Developing deep group reflection within a Critical Reflection Action Learning set. Action Learning: Research and Practice, 13(3). https://doi.org/10 .1080/14767333.2016.1220176

Stewart, D. W., \& Shamdasani, P. N. (2014). Focus Groups: Theory and Practice. Thousand Oaks, Calif.: Sage Publications.

Švarc, J., \& Dabić, M. (2015). Evolution of the Knowledge Economy: a Historical Perspective with an Application to the Case of Europe. Journal of the Knowledge Economy, 1-18. https://doi.org/10.1007/s13132-015-0267-2

Tadajewski, M. (2016). Focus groups: history, epistemology and non-individualistic consumer research. Consumption Markets and Culture, 19(4). https://doi.org/10.1080/1 0253866.2015 .1104038

Tobón, S. (2013). Formación integral y competencias. Pensamiento complejo currículo didáctica y evaluación (4a.). Bogotá: ECOE.

UNESCO. (1998). Declaración Mundial sobre la Educación Superior en el Siglo XXI: Visión y Acción.

Vaca, J., Aguilar, V., Gutiérrez, F., Cano, A. y Bustamante, J. (2015). ¿Qué demonios son las competencias? Aportaciones del constructivismo clásico y contemporáneo. México: Universidad Veracruzana.

Villa, A., \& Poblete, M. (2008). Competence Based Learning. A Proposal for the assessment of generic competences. Bilbao: University of Deusto.

Weber, A. S. (2011). The role of education in knowledge economies in developing countries. Procedia - Social and Behavioral Sciences, 15, 2589-2594. https://doi. org/10.1016/j.sbspro.2011.04.151 


\section{ANEXOS}

\section{ANEXO A}

\section{Ejemplos de codificación abierta}

\begin{tabular}{|l|l|}
\hline \multicolumn{1}{|c|}{ Transcripción } & \multicolumn{1}{|c|}{ Codificación } \\
\hline $\begin{array}{l}\text { Hay un tema importante, que está fallando y son los valores del } \\
\text { personal. Estamos hablando de una persona que va a salir de } \\
\text { egresado desde ahí, pero finalmente no necesariamente está muy } \\
\text { bien formado. No queremos que se queden en temas técnicos } \\
\text { porque al final esperamos que lleguen a directivos y para eso } \\
\text { deben de trabajar. }\end{array}$ & \\
\hline Compleador \\
valiosa que ha salicamos egresados de esta carrera, hay gente muy \\
ocupar cualquier puesto. Entonces, es más bien cómo les damos \\
la experiencia, cómo a muchos nos ha tocado ¿no?, empezar \\
desde arriba, pero precisamente que vayan creciendo dentro de \\
la misma empresa, porque nadie sale sabiendo que en Santander \\
se trabaja de esta manera. O sea, en la escuela te dan las bases, \\
lo que realmente uno llega a ejecutar es dentro de la empresa \\
cuando la conoces. Entonces, creo que no debemos de limitar el \\
puesto sino pulirlo, darle herramientas. Si la persona es capaz, va a llegar. \\
a llegar.
\end{tabular}




\section{ANEXO B}

\section{Dos ejemplos de memorandum}

\begin{tabular}{|c|c|c|}
\hline \multicolumn{3}{|c|}{ Memorándum al grupo de enfoque } \\
\hline No. 14 & Fecha: 05/02/2016 & Código: Proactividad deficiente \\
\hline \multicolumn{3}{|c|}{$\begin{array}{l}\text { Culturalmente los empleados tienen el hábito de no hacer más de lo que les corresponde para que no } \\
\text { les asignen más trabajo, y esa es la mentalidad con la que llegan. En general es un problema cultural, } \\
\text { en varias ocasiones dicen los egresados que mejor no hablan porque en cuanto comentan una idea, } \\
\text { de inmediato se convierte en su responsabilidad ponerla en práctica, con el mismo sueldo, puesto, } \\
\text { horario, recursos, etc., entonces han aprendido que mejor no abren la boca. }\end{array}$} \\
\hline ccion: Ident & & \\
\hline
\end{tabular}

\section{Memorándum al grupo de enfoque}

\begin{tabular}{l|l|l} 
No. 19 & Fecha: 05/02/2016 & Código: Necesitan comunicación asertiva
\end{tabular}

De acuerdo con lo que manifestaron los empresarios, los estudiantes salen con deficiencias muy grandes en comunicación oral y escrita, no saben elaborar de manera correcta un escrito y no saben expresarse oralmente en una reunión de trabajo, con lo que los perciben muy inseguros. Si bien es cierto, es un problema que traen desde niveles anteriores, no se puede cerrar los ojos ante algo tan evidente y que los alumnos sufren las consecuencias al salir de la universidad. Considero que esto sí es responsabilidad de la universidad, por lo que se debe revisar lo que se está haciendo en las materias de Expresión oral y escrita, e implementar acciones para corregir esta situación.

Acción: La expresión oral correcta es una competencia profesional genérica y, si la universidad no está cumpliendo su objetivo al respecto, las personas que sí logran remontar esa deficiencia ¿cómo lo hacen? 